\title{
The alteration of intellectual performance after lateralized brain-injury in man
}

\author{
PAUL SATZ, WAYKE RICHARD ${ }^{1}$ AKD AUBREY DAMIELS?
}

UNIVERSITY OF FLORIDA

The effects of left-and right-brain lesions on verbal (VIQ) and performance (PIQ) subtests of the WAIS were investigated. Multivariate covariance analysis was used to statistically adjust for effects of age, education and intelligence. Results showed a decrement on VIQ scores with left-sided lesions. Right-sided lesions, however, did not reveal the expected impairment on "nonverbal" PIQ scores.

The present study represents a reexamination of the relationship between left and right hemisphere lesions on the Verbal (VIQ) and Performance (PIQ) Scales of the Wechsler Adult Intelligence Scale (Wechsler, 1958). Factor analytic studies of the earlier Wechsler-Bellevue Scale (Cohen, 1952) found significant loadings on two primary factors, a verbal organization (VIQ) and a nonverbal organization factor (PIQ). Reitan (1955) has suggested that these composite Wechsler scales vary with lesion laterality, with left hemisphere lesions associated with greater impairment on the verbal subtests (VIQ) and right hemisphere lesions associated with greater impairment on the nonverbal performance subtests (PIQ).

Similar dissociation effects have been observed between left and right brain lesions using additional measures of "verbal" and "nonverbal" performance which, in turn, have led some investigators to postulate a degree of functional independence with respect to hemispheric function in man (Milner, 1962).

The alteration on tests of verbal reasoning after left hemisphere injury is not surprising in view of the marked incidence of left cerebral dominance for speech in man (Penfield \& Roberts, 1959). On the other hand, the postulation of a separate "dominance" for nonverbal perceptual organization in the right hemisphere is less clearly inferred from the data. Some tests of nonverbal reasoning have shown similar impairment after either left- or right-sided injury (Heilbrun, 1956), while other "nonverbal" tests have revealed nonspecific effects, i.e., impairment independent of locus or laterality of lesion (Satz, 1966; Teuber \& Weinstein, 1956). Two general problems probably account for these discrepancies. First, some investigators have made premature attempts to extrapolate from test data to concepts of brain localization without first proceeding to a more careful analysis of abilities measured by the tests. Second, design of the studies has often failed to control for more than one concomitant variable in the analysis of a given experimental variable. The reason is that it is often impossible or impractical to control for additional concomitant variables by direct selection of the Ss, particularly with braininjured groups. The possibility therefore exists that differences among treatment means may be accounted for by chance differences in the age, education or intelligence of the Ss. The present study was addressed primarily to the latter problem and employed the method of multivariate covariance analysis (Lindquist, 1953) to obtain statistical control over several concomitant variables in the determination of verbal (VIQ) and performance (PIQ) alterations after brain-injury in man. Subjects

Ss were assigned to one of six different treatment groups on the basis of psychiatric, neurological and laboratory examination. The first four groups were represented by different categories of lateralized brain-injury, including left-sided lesions ( $\mathrm{LH}, \mathrm{N}=\mathbf{2 3}$ ), right-sided lesions ( $R H, N=24$ ), nonspecific lesions (NS, $\mathrm{N}=33$ ) and bilateral lesions ( $\mathrm{BL}, \mathrm{N}=23$ ). The composition of these groups was equated for type of lesion and consisted of vascular, neoplastic, traumatic and convulsive disorders. Ss were all right-handed. The remaining two groups were composed of medicalpsychiatric cases (MP, $\mathrm{N}=114$ ) and normal controls (NO, $\mathrm{N}=51$ ).

\section{Statistical Analysis and Hypotheses.}

Two analyses of covariance were computed for VIQ and PIQ separately, using an IBM 709 computer (UCLA BIMED Program, BMD 04V). ${ }^{3}$ In the first analysis, VIQ was used as the criterion variable and was adjusted for the effects of the following three concomitant variables: (1) age, (2) education, and (3) PIQ. In the second analysis, adjustments were made on PIQ criterion scores against the following three concomitant variables: (1) age, (2) education, and (3) VIQ. Two hypotheses were examined and were based upon the preceding two analyses: Hypothesis 1: Adjusted VIQ scores would be lower in the unilateral left hemisphere group than in the remaining neurological and control groups. Hypothesis 2: Adjusted PIQ scores would be lower in the unilateral right hemisphere group than in the remaining neurological and control groups.

\section{Results and Discussion}

Two general findings emerged. The analysis of covariance of VIQ was significant $(F=8.45$, $\mathrm{df}=5 / 259$, $p<.001)$ and disclosed an overall difference between criterion groups. Analysis of the individual means 
further demonstrated that the adjusted scores on VIQ were significantly lower in the group with left hemisphere injury (LH) than in any of the other treatment groups (Hypothesis 1). The adjusted VIQ means wereas follows: $\mathrm{LH}=92.30, \mathrm{RH}=106.65, \mathrm{NS}=100.46, \mathrm{BL}=98.56$, $\mathrm{MP}=104.27$, and $\mathrm{NO}=107.15$. The analysis of covariance of $\mathrm{PIQ}$ was also significant $(\mathrm{F}=3.53, \mathrm{df}=5 / 259$, $\mathrm{p}<.01)$. However, when individual means were compared, it was found that the adjusted PIQ. scores were not significantly and uniformly lower in the right hemisphere groups (Hypothesis 2). The only difference was between the RH and LH groups, with lower PIQ values associated with the $R H$ group $(t=4.65, p<.001)$. The adjusted $\mathrm{PIQ}$ means were as follows: $\mathrm{LH}=104.92$, $\mathrm{RH}=96.13, \mathrm{NS}=95.84, \mathrm{BL}=94.70, \mathrm{MP}=99.37$, and $\mathrm{NO}=$ 101.16 .

The present findings suggest that lesions involving the left hemisphere in man lead to alterations in verbal reasoning as measured by WAIS verbal subtests (VIQ). Furthermore, the impairment in verbal performance was not simply a function of aphasia in that only two Ss in the left-sided group had a clinical history of language disorder. On the other hand, lesions involving the right hemisphere failed to show a consistent alteration on the "nonverbal" performance subtests (PIQ). Comparisons between the $\mathrm{RH}$ group and the non-lesion controls (MP and NO) on PIQ were not significantly different. Thus, the hypothesis of a relationship between right hemisphere injury and impaired "nonverbal" intelligence was not supported in this study. If one assumes that the WAIS Performance Scale (PIQ) is a valid estimate of "nonverbal" intellect, then one might conclude that previous reports of impaired performance on this scale, after right-sided injury, were probably due to chance differences associated with uncontrolled concomitant variables.

\section{References}

Cohen, J. Factors underlying Wechsler-Bellevue performance of three neuropsychiatric groups. J. abnorm. soc. Psychol., 1952, 47, 359-365.

Heilbrun, A. B. Psychological test performance as a function of lateral localization of cerebral lesion. J. comp. physiol. Psychol., 1956, 49, 10-14.

Lindquist, E. F. Design and analysis of crperiments in psychology and cducation. Boston: Houghton Hifflin, 1953.

Milner, Brenda. Laterality effects in audition. In V. B. Mountcastle (Ed.), Interhemispheric relations and cerebral dominance. Baltimore: Johns Hopkins Press, 1962. Pp. 264-277.

Reitan, R. M. Psychological deficit. Annu. Rev. Psychol., 1962. $13,415-444$.

Satz, P. Specific and nonspecific effects of brain lesions in man. J. abnorm. Psychol., 1966, 71, 65-70.

Teuber, H. L., \& Weinstein, S. Ability to discover hidden figures after cerebral lesions. Amer. med. Assoc. Arch. Neurol. Psychiat., 1956, 76, 369-379.

Wechsler, D. The measurement and appraisal of adult intelliqence. (4th ed.) Baltimore: Waverly, 1958.

\section{Notes}

1. Now at George Peabody Teachers College.

2. Now at Georgia Mental Health Institute.

3. Data were analyzed at the Computing Center, University of Florida. 